\section{Revista de la \\ Universidad del Thulia}

Fundada en 1947 por el Dr. Jesúns Enrique Lossada

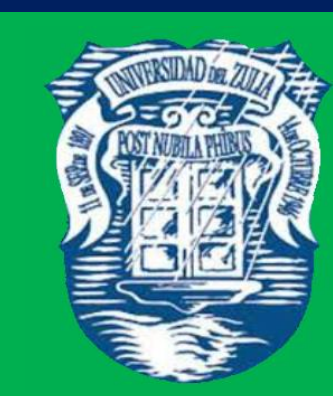

Ciencias del

Algreo

Ingemieria

y Teemología

\section{Aกัต 11 No 29}

Enero - Abril 2021

Tercera Época

Maracaibo-Venezuela 


\title{
Investigation about electrostatic three-wall carbon nanotubes (MWCNT), including doping with BN: a model for the nano capacitor
}

Nabieh Farhami*

Majid Monajjemi** $^{* *}$

\begin{abstract}
Three-walled boron \& nitride nanotubes are used for a theoretical study of a cylindrical molecular capacitor, including an inner cylinder with a positive charge distribution and an outer cylinder with a negative charge distribution. Due to the semiconductor characteristic and dielectric functionality of SWBNTs, DWBNTs and TW (B\&C) NTs can be used as a capacitor. Although the SWBNTs @ SWCNTs behave like Nano cylindrical capacitors, we have shown in this study that a dopant of $\mathrm{BN}$ in the inner cylinder reduces the energy gap and yields a better capacitance. The dopant of BN in the outer cylinder results in an inverse charge distribution (outer is positive and inner is negative). Therefore under these circumstances, the term capacitor would be meaningless, thought, the gap and the interaction energy decreases compared to the non-dopant form of those capacitors. Density functional theory (DFT) calculations have performed for the structure and stability of three wall carbon Nano tubes (TW (BN\&C) NTs). In this work, it was calculated the geometrical structure, and stability to predict NMR and thermodynamics parameters. A mixing of SWBNNTs @ DWCNTs has been modeled and calculated for the suitable structures to storage the $\mathrm{H}_{2}$ molecules for increasing the dielectric. We have found these kinds of Nano-structures are useful for maximum storages of charges compare to other cylindrical capacitor.

KEYWORDS: three wall carbon nanotubes (TWCNTs), BN doping, Nano capacitor, cylindrical molecular capacitor.
\end{abstract}

*Department of Chemistry, Science and Research Branch, Islamic Azad University, Tehran.

${ }^{* *}$ Department of chemical engineering, Central Tehran Branch, Islamic Azad University, Tehran, Iran: Maj.monajjemi@iauctb.ac.ir

Recibido: 23/01/2020

Aceptado: 19/02/2020 


\section{Investigación acerca de la electrostática de tres nanotubos de carbono de pared (MWCNT), incluido el dopaje con BN: un modelo para el condensador nano}

RESUMEN

Los nanotubos de boro y nitruro de tres paredes se utilizan para un estudio teórico de un condensador molecular cilíndrico, que incluye un cilindro interno con una distribución de carga positiva y un cilindro externo con una distribución de carga negativa. Debido a la característica semiconductora y la funcionalidad dieléctrica de los SWBNT, los DWBNT y los TW (B\&C) NT se pueden usar como condensadores. Aunque los SWBNTs \& SWCNTs se comportan como condensadores cilíndricos nano, hemos demostrado en este estudio que un dopante de BN en el cilindro interno reduce la brecha de energía y produce una mejor capacitancia. El dopante de BN en el cilindro externo da como resultado una distribución de carga inversa (el exterior es positivo y el interior es negativo). Por lo tanto, en estas circunstancias, el término condensador no tendría sentido, aunque el espacio y la energía de interacción disminuyan en comparación con la forma no dopante de esos condensadores. Los cálculos de la teoría funcional de la densidad (DFT) se han realizado para la estructura y la estabilidad de los tubos nano de carbono de tres paredes (NT (BN\&EC) NT). En este trabajo, se calculó la estructura geométrica y la estabilidad para predecir los parámetros de RMN y termodinámica. Se ha modelado y calculado una mezcla de SWBNNTs@DWCNTs para las estructuras adecuadas a objeto de almacenar las moléculas de H2 para aumentar el dieléctrico. Hemos encontrado que este tipo de nanoestructuras son útiles para el almacenamiento máximo de cargas en comparación con otros condensadores cilíndricos.

PALABRAS CLAVE: nanotubos de carbono de tres paredes (TWCNT), dopaje BN, condensador nano, condensador molecular cilíndrico.

\section{Introduction}

Although much study has been done for nanotube carbon phenomenon, there are a few studies for MWCNTs such as TWCNTs (Bourgeois et al., 2000). The carbon nanotube (CNT) is a representative Nano-material. CNT is a cylindrically shaped carbon material with a Nano-metric-level diameter. Its structure, which is in 
the form of a hexagonal mesh, resembles a graphite sheet and it carries a carbon atom located on the vertex of each mesh. The sheet has rolled and its two edges have connected seamlessly (Iijima Sumio, 1991).

Although it is a commonplace material using in pencil leads, its unique structure causes it to present characteristics that had not found with any other materials. CNT can be classified into single-wall CNT, double-wall CNT and multiwall CNT according to the number of layers of the rolled graphite (Iijima Sumio, 1991). The type attracting most attention is the single-wall CNT, which has a diameter deserving the name of "nanotube" of 0.4 to 2 nanometers. The length is usually in the order of microns, but single-wall CNT with a length in the order of centimeters has recently released (Frackowiak, Béguin, 2002). CNT can be classified into single-wall CNT, double-wall CNT and multi-wall CNT according to the number of layers of the rolled graphite. The type attracting most attention is the single-wall CNT, which has a diameter deserving the name of "nanotube" of 0.4 to 2 nanometers (Bourgeois et al., 2000).

The length is usually in the order of microns, but single-wall CNT with a length about centimeters have recently released. The extremities of the CNT have usually closed with lids of the graphite sheet. The lids consist of hexagonal crystalline structures (six-membered ring structures) and a total of six pentagonal structures (five-membered ring structures) placed here and there in the hexagonal structure (Monajjemi et al., 2011). The first report by Iijima was on the multiwall form, coaxial carbon cylinders with a few tens of nanometers in outer diameter. Two years later, up to now, single walled nanotubes were reported in various works (Iijima Sumio, 1991). SWCNTs have considered as the leading candidate for Nano-device applications because of their one-dimensional electronic bond structure, molecular size, and biocompatibility, controllable property of conducting electrical current and reversible response to biological reagents hence SWCNTs make possible bonding to polymers and biological systems such as DNA and carbohydrates. Boron nitride 
nanotube (BNNTs) has attracted many interests due to their large gap semi conducting character. Boron nitride (BN) is a structural existing in cubic (diamondlike), hexagonal (graphite-like), turbo static, and amorphous forms these compounds have been produced by a variety of methods, such as arc melting, high temperature chemical reaction, carbon nanotube templates, and laser ablating. The most attention has been focused on the development of new methods for the production of nanotube and inorganic fullerene of other materials (Lee et al., 2009).

In addition, theoretical calculations have been described the possible existence of small BN clusters. Theoretical studies have been performed for BN doped in CNTs which it has been found that a structure built from squares and hexagons is more stable than those built from pentagons and hexagons. This is because in the second case less stable B-B and N-N bonds are formed (Derakhshandeh, 2015). The most stable TWCNTs structure is built from CNTs doping with BN. In this work, we focused on TWCNTs and TWC (BN) NTs Nano-con. Our aim was to obtain the global minimum energy structure (Fig.1). For this structure, we use the hybrid B3LYP exchange-correlation functional within density functional theory. Primary, structure optimization calculated and then Nuclear Magnetic Resonance (NMR) parameters by density Functional Theory (DFT) method calculated on the optimized structure. In material sciences Boron nitride, which appears in a manifold of crystalline modifications, has been an extremely practical material with hexagonal and cubic boron nitride as most outstanding materials (for doping). The BN cluster is a polar molecule and BN doped in nanotubes have an inert chemical structure. We can see that there is a negative charge at nitrogen atom and a positive charge at boron atom, so we can use an electrophilic or nucleophilic reagent as a solution for BN clusters (Farhami et al., 2017).

$\mathrm{BN}$ nanotubes are very suitable for composite materials because these structures have a higher temperature resistance to oxidation than the carbon nanotubes. All the BN nanotubes are semiconductors. The BN doped in nanotubes 
have the band gaps which can be greater than $2 \mathrm{eV}$ for most tubes also we know that the smallest carbon nanotubes are semiconductor and these structures obtain the properties of graphite when the diameter of these structures increases but BN nanotubes are semiconductors without attention to the diameter. On the basis of the similarities in characteristics between carbon and $\mathrm{BN}$-based ( $\mathrm{BN}=$ boron nitride) substances, BN-based nanotubes can be stable and therefore their electronic structure can be studied. The comparison between BN nanotubes and carbon nanotubes shows that BN nanotubes have more interesting characteristics for doping in carbon nanotubes. Recently the mixing of boron nitride (BNNTs) and (CNTs) in a nanoscale particles have been investigated and these structures are made up of conical shells without any seamless. Most of the studies about these compounds have been done so far with carbon structures. Considering the above mentioned, (BN-C) NTs nanotubes are very important and interesting for new research, especially for $\mathrm{H} 2$ storage and can open a huge spectrum in the field of theoretical and experimental research. In the Fig.l structure of TWCNTs is shown and this particular nanometer configuration has been proposed in this research (Madani et al., 2017).

\section{Theoretical background}

Our system is similar to narrow seamless graphitic carbon nanotube cylinders, showing an unusual combination of a nanometer-size diameter and millimeter-size length. This topology, combined with the absence of defects on a macroscopic scale, gives rise to uncommon electronic properties of individual single-wall nanotubes which can be metallic, semiconducting or insulating, depending on their diameter and chirality.

Consider a cylindrical capacitor of length " $d_{l}$ ", inner radius " $R_{i n n}$ ", outer radius " $R_{\text {out }}$ ", and charge $\mathrm{Q}=d_{l} \cdot q_{\lambda}$ which $q_{\lambda}$ is the charge per unit length (magnitude) on each cylinder (Schemel). Assume " $d_{l}$ " $\gg$ " $R_{\text {out }}$ " and Neglect fringing and electric field between cylinders: use Gauss' law $E\left[2 \pi r d_{l}\right]=\frac{d_{l} \cdot q_{\lambda}}{\varepsilon_{0}} \Rightarrow E(r)=\frac{q_{\lambda}}{2 \pi \varepsilon_{0} r}$ (1) and 
electric potential between cylinders: use $\mathrm{V}_{\text {out }}=0 \Rightarrow \mathrm{V}(\mathrm{r})=-\int_{R_{\text {inn }}}^{r} E(r) d r=\frac{-q_{\lambda}}{2 \pi \varepsilon_{0}} \int_{o}^{r} \frac{d r}{r}=$ $\frac{-q_{\lambda}}{2 \pi \varepsilon_{0}} \ln \frac{r}{R_{\text {inn }}}(2)$

And $V=V_{+}-V_{-}=V_{(i n n)}-V_{(o u t)}=\frac{Q}{2 \pi \varepsilon_{0} L} \ln \frac{R_{o u t}}{R_{\text {inn }}}$ (3) and capacitance for cylindrical geometry is: $\bar{C}_{g} \equiv \frac{Q}{V}=\frac{2 \pi \varepsilon_{0} d_{l}}{\ln \frac{R_{\text {out }}}{R_{\text {inn }}}} \quad$ (4). Where $\Delta V_{(i n n-o u t)}$, is a positive quantity because $2 k \ln \left(\frac{R_{\text {out }}}{R_{\text {inn }}}\right)$ is a positive quantity. This is due to the fact that the outer layer is at a higher potential than the inner layer. In our system the calculation of the Nanocylindrical capacitors can be obtained from the electrical potential $\varnothing(r, Z)$ in the space between two coaxial cylinders of radii $R_{\text {inn }}$ and $R_{\text {out }}$ finite length $d_{l}$ in the $z$ direction $\left(0 \leq z \leq d_{l}\right)$. We have assumed that the geometrical capacitance in our system is a function of $d_{l}, R_{\text {inn }} R_{\text {out }}$ or $C_{g}=F\left(d_{l}, R_{\text {inn }}, R_{\text {out }}\right)$.

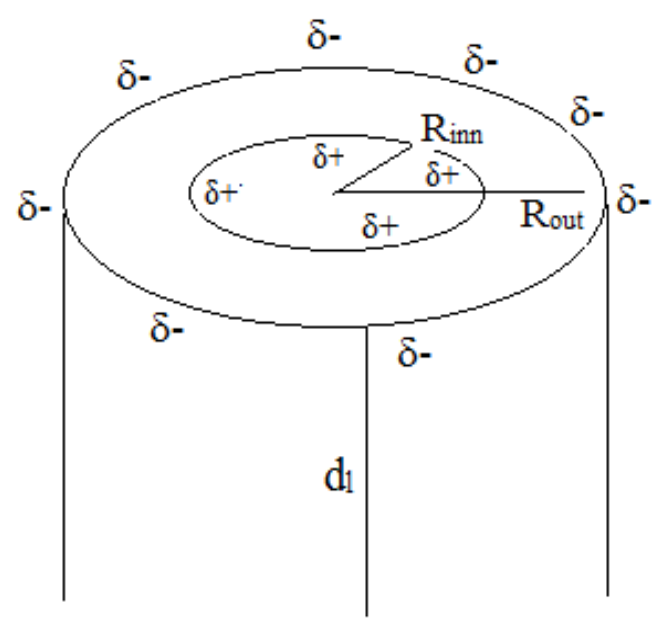

Scheme 1: A schematic of a cylindrical Nano-capacitor

The capacitance in our model can be calculated via $C_{g}=$ $\frac{2 \pi R_{i n n} \varepsilon_{0}}{V} \int_{0}^{d_{l}}\left(\frac{\partial \emptyset}{\partial r}\right)_{r=R_{i n n}} d z$ (5) in a finite nanometer-scale cylindrical capacitor (based on the classical electrodynamics). However, on very small scales the quantum corrections will appear. It is known that typical inhomogeneous electric fields used in the polarizability measurements for metallic clusters are in the order of $10^{5} \mathrm{~V} \mathrm{~cm}^{-1}$ 
and field gradients are approximately $10^{5} \mathrm{~V} \mathrm{~cm}^{-2}$. These fields induce a dipole moment and slightly deformed clusters. Therefore, to strictly preserve the shape of a nanometer-scale capacitor, weaker and homogeneous fields should be applied in electronic devices. To calculate the capacitance in eq.4 and eq.5 for $\left(\bar{C}_{g}, C_{g}\right)$, the potential difference applied between two cylindrical plates $\mathrm{V}=V_{(\text {inn })}-V_{(\text {out })}$ has been calculated by the total different of atomic point charges versus the bond distances. The original total DOS (TDOS) of this system has been calculated based on, $\operatorname{TDOS}(E)=\sum_{i} \delta\left(E-\varepsilon_{i}\right)(6)$. Where the eigenvalue " $\varepsilon$ " is set of single-particle Hamilton and " $\delta$ " is the Dirac delta function which can be yielded after replacing Gaussian. There is another Gaussian function as: $G(X)=\frac{1}{c \sqrt{2 \pi}} e^{\frac{-x^{2}}{2 c^{2}}}$ Which the FWHM $\left(c=\frac{F W H M}{2 \sqrt{2 \ln 2}}\right)$ stands for "full width at half maximum", an adjustable parameter in multi-wave function program where the larger FWHM results in a smoother looking TDOS graph, making the analysis to be performed easier. The normalized Lorentzian function is defined as $L(X)=\frac{F W H M}{2 \pi} \frac{1}{x^{2}+0.25 F W H M^{2}}$ Pseudo-Voigt function is weighted as linear combination of Gaussian function and Lorentzian function $P(x)=w_{\text {gauss }} G(x)+\left(1-w_{\text {gauss }}\right) L(x)$. The curve map of broadened partial DOS (PDOS) and overlap DOS (OPDOS) are valuable for visualizing orbital composition analysis PDOS function of fragment which $A$ is defined as $\operatorname{PDOS}_{A}(E)=$ $\sum_{i} \Xi_{i, A} F\left(E-\varepsilon_{i}\right)(7)$ where $\Xi_{i, A}$ is the composition of fragment "A" in orbital $i$. The OPDOS between fragment "A" and "B" is defined asOPDOS ${ }_{A, B}(E)=\sum_{i} X_{A, B}^{i} F\left(E-\varepsilon_{i}\right)$ (8) where $X_{A, B}^{i} A, B$ is the composition of total cross term between fragment $A$ and $B$ in orbital. Both original and broadened TDOS/PDOS/OPDOS are shown in this study. It is important to note that the height is only meaningful for lines (original data) and not for curves and left-axis and right-axis correspond to TDOS/PDOS and OPDOS respectively (Lu, Chen, 2012). 


\section{Computational details}

Calculations were performed using GAMESS-US packages as well as some other parallel ab-initio and molecular mechanics programs. In this study, we have mainly focused on getting the optimized structures for each tube from DFT methods including the m05 and m06 groups. The m062x, m06-L, and m06-HF are a novel Meta hybrid DFT functional with a good correspondence in non-bonded calculations and are useful for calculating the energies of the distance between two coaxial cylinders of radii $R_{\text {inn }}$ and $R_{\text {out }}$ in the cylindrical capacitor. ONIOM methods including 3 levels of 1-high calculation (H), 2-medium calculation (M), and 3-low calculation (L) have been performed in our study. The ab-initio and DFT methods are used for the model system of the ONIOM layers and the semi empirical methods of Pm6 (including pseudo=lanl2) and Pm3MM are used for the medium and low layers, respectively. The semi empirical methods have been used in order to treat the non-bonded interactions between two parts of SW-BN-NTs and SWCNTs cylinders. M06 and m06-L (DFT) functional are based on an iterative solution of the Kohn-Sham equation from density functional theory in a plane-wave set with the projector-augmented wave pseudo-potentials.

The Perdew-Burke-Ernzerhof (PBE) exchange-correlation (XC) functional of the generalized gradient approximation (GGA) has also been used. The optimizations of the lattice constants and the atomic coordinates are made by the minimization of the total energy. It is interesting to note that the tubular morphology of SW-BN-NTs is fundamentally different from the usual tubular forms of carbon and boron nitride (BN) nanotubes. In the outer shell, the more electronegative atoms ( $\mathrm{N}$ atoms) are pushed radially outward and the more electropositive atoms (Ga atoms) are displaced inward. In the inner shell, the opposite phenomenon occurs and the Ga atoms move outward while the $\mathrm{N}$ atoms move inward. This result for BN nanotubes is expected since the overlap between the $\mathrm{B}$ and $\mathrm{N}, \mathrm{PZ}$ orbitals is weaker. According to the Mulliken population analysis, a considerable amount of electron charge is 
transferred from $\mathrm{Ga}$ to $\mathrm{N}$, resulting in an ionic bonding nature. $\mathrm{N}$ loses some $\mathrm{s}$ electrons and gains more pelectrons, whereas Ga loses more $p$ than s electrons. The average B-N bond length in the optimized structure of SW-BN-NTs in our calculation is between 1.9-1.945 $\mathrm{A}^{\circ}$, which is shorter than the bond length of $1.95 \mathrm{~A}^{\circ}$ in the crystal structure of Wurtzite. A fixed SW-BN-NTs geometry with BN bond lengths of $1.45 \AA$ is chosen with no further geometry optimization.

The outer ring, initially placed at the center of the inner tube, is rigidly axially been shifted and rotated around the fixed inner shell. At each inter-tube configuration, a single-point calculation is carried out and the total energy is recorded. The resulting sliding-rotation energy surfaces are used to fix our model in a better position. We employed density functional theory with the van der Waals density functional to model the exchange-correlation energies of SW-BN-NTs. The double $\zeta$-basis set with polarization orbitals (DZP) were used for tubes. For noncovalent interactions, the B3LYP method is unable to describe the van der Waals interactions, e.g., the capacitor systems by medium-range interactions such as the interactions of two cylinders. The B3LYP and most other functional are rather insufficient to illustrate the exchange and correlation energy for distant non-bonded medium-range systems. Moreover, some recent studies have shown that inaccuracy for the medium-range exchange energies leads to large systematic errors in the prediction of molecular properties. Therefore, we further calculated the interaction energy between two coaxial cylinders of radii $R_{\text {inn }}$ and $R_{\text {out }}$ for each tubes in our structures (The number of nanotubes was varied to examine size-dependent electrical properties) using other ab-initio method. The interaction energy " $\Delta E_{S}$ " between two cylinders of the capacitor has been calculated via an Mp6 method based on the eq.6. $\Delta E_{S}(\mathrm{eV})=E_{\text {total }}-\left(E_{S W-G a N-N T(\text { inn })}+E_{S W C N T(\text { out })}\right)$ (9) (Table 1).

The charge transfer and the electrostatic potential-derived charges were also calculated using the Mullikan charge distribution the representative atomic charges for molecules should be computed as average values over several molecular 
conformations. A detailed overview of the effects of the basis set and the Hamiltonian on the charge distribution can be found in previous studies. We have also extracted the charge density profiles from the first principle calculations through an averaging process. DFT (density functional theory) is one of the computational methods which can be used in different systems and it is more useful for some calculations than other methods. It is clear that basis sets are vast various. The Gaussian 98 program was run to obtain the best prediction of this particular structure. Also all Ab-Initio and DFT (density functional theory) calculations were done with the Gaussian 98 program. Frequency analyses were carried out to show that the optimized structures are true minima or transition states on the potential energy surfaces of a specific structure without imaginary frequencies. In this work, geometry optimizations in the gas phase for TWCNTs were performed at density functional theory (DFT) level with B3LYP and Ab-Initio with HF methods in different basis sets at the temperature of 298.15K.

The parameters were calculated for TWCNTs in the gas phase in different methods and basis sets include thermodynamic and NMR parameters. The chemical shielding shows the phenomenon which is dependent on the secondary magnetic field which is built by the induced movements of the electrons which encompass the nuclei. The chemical shielding is built by a three-by-three matrix which is biodegraded into a single scalar term, three antisymmetric pseudo vector components, and five components which correspond to a symmetric tensor. It can be observed the single scalar and the five symmetric tensor elements in the normal NMR spectra of the solids. The chemical shielding tensor includes the chemical shift isotropy (CSI) and chemical shift anisotropy (CSA) and the anisotropy $(\Delta \sigma)$ of the tensor, the shielding tensor asymmetry parameter $(\eta)$ and chemical shift $(\delta)$ are calculated. The thermodynamic parameters that were calculated in this research are Gibbs free energy, enthalpy, internal energy (It is clear that the sum of zero point energy (ZPE) and thermal energy is internal energy.) and entropy then these reports 
were compared with each other in order to obtain the best results. These results were reported in tables.

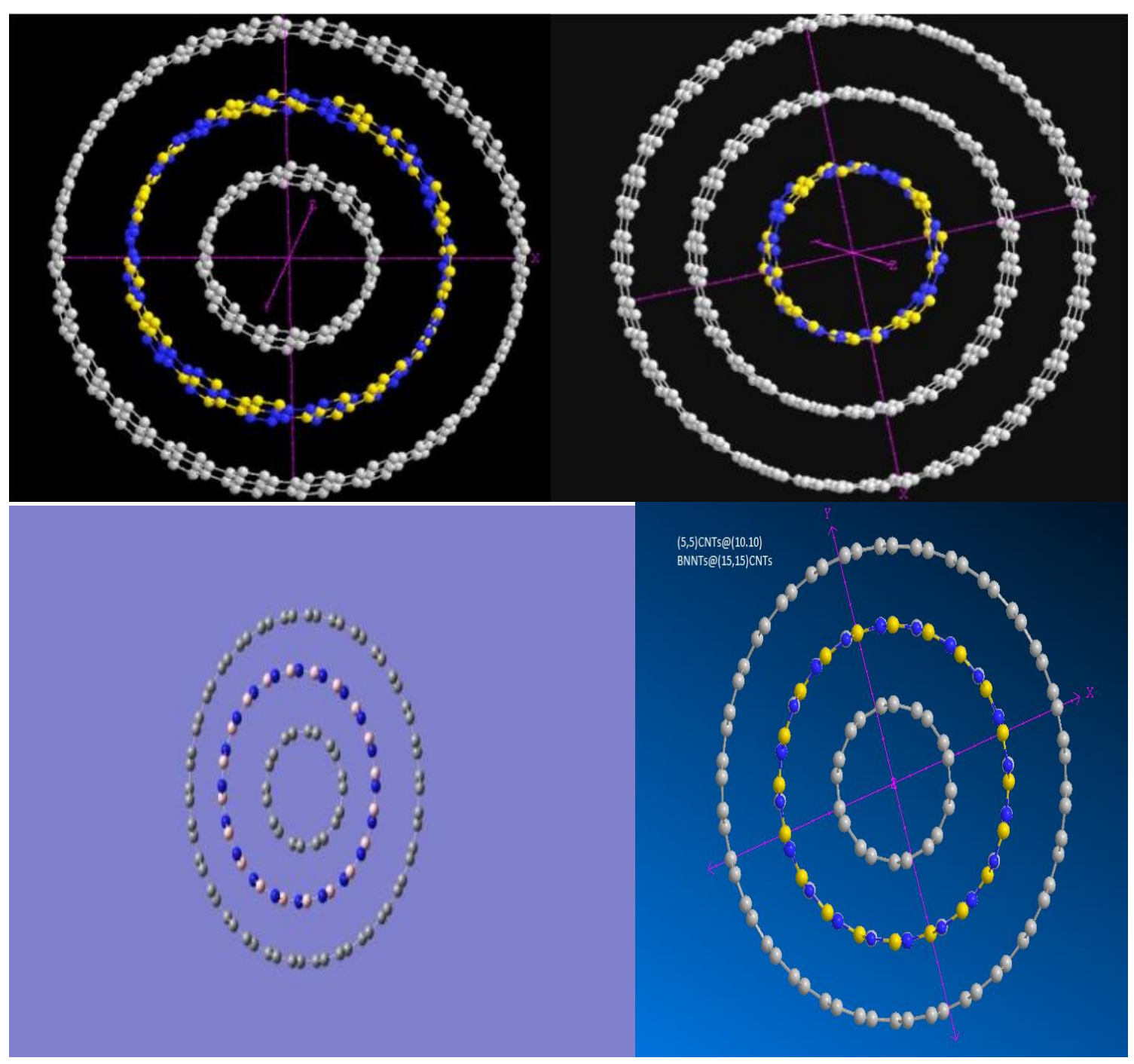

Fig.l: Optimized of Various TW(C\&BN)NTs

\section{Results and Discussion}

The 3D BN tubes in various diameter and chirality are designed for finding a stable form inside the SWCNTs. The stability depends on the distance between inner 
radius " $R_{\text {inn }}$ ", and outer radius " $R_{\text {out }}$ in one hand and the chirality on other hand Table 1.

Energies and HOMO LUMO for several capacitors are listed in Table 1.
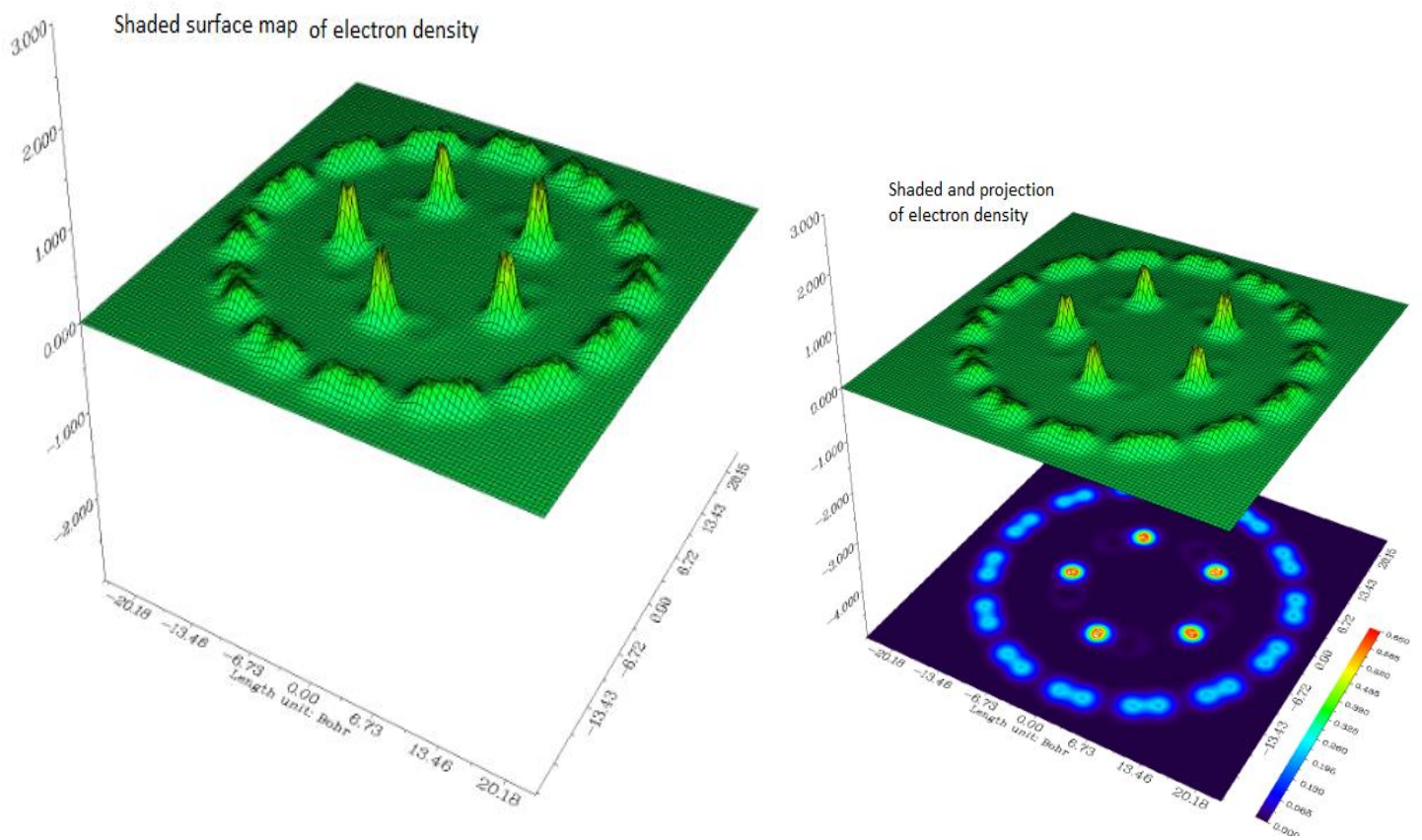

Fig. 2: Shaded surface map of cylindrical capacitor

Table 1 . The band structure, Fermi level energy, stability and relative energy of the $C$ $(\mathrm{m}, \mathrm{m})$ NTs @ C (n, n)NTs structures, including 7 molecule of BN dopant in outer cylinder. And 3 molecule of BN dopant in inner cylinder.

\begin{tabular}{|c|c|c|c|c|c|c|c|}
\hline CNTs@CNTs & $\begin{array}{l}\text { Fermi } \\
\text { energy }\end{array}$ & HOMO & LUMO & $\begin{array}{l}\text { Gap } \\
\text { energies }\end{array}$ & $\begin{array}{l}\text { Relative } \\
\text { energies }\end{array}$ & $\begin{array}{l}\text { Stability } \\
\text { energy }\end{array}$ & $\begin{array}{l}\mathrm{R}(\text { in) } \\
\mathrm{R} \text { (out) }\end{array}$ \\
\hline$(4,4) @(9,9)$ & -814.2 & -0.310109 & -0.215009 & 249.68 & - & $-5.53 \mathrm{eV}$ & 3.58 \\
\hline$(5,5) @(10,10)$ & -901.7 & -0.34345 & -0.228012 & 303.15 & +0.057903 & $-0.69 \mathrm{eV}$ & 4.53 \\
\hline$(3,3) @(7,7)$ & -913.5 & -0.34795 & -0.240903 & 281.06 & 1.9068974 & $-4.39 \mathrm{eV}$ & 3.58 \\
\hline$(4,4) @(11,11)$ & -905.6 & -0.34494 & -0.227262 & 308.98 & 0.0000000 & $+0.25 \mathrm{eV}$ & 6.37 \\
\hline$(5,5) @(12,12)$ & -850.9 & -0.324091 & -0.227069 & 254.72 & +0.522609 & $+10.00 \mathrm{eV}$ & 6.39 \\
\hline
\end{tabular}


The differences in the band structure and Fermi level energy of different tubes have been calculated. Furthermore, we have presented the number of states in unit energy interval through density of states (DOS) (Table 1 and Figs.2 \& 3). Since the energy levels are contiguous and our system is isolated (the energy levels are discrete), DOS was plotted as a curved map and we have considered those graphs as a tool for analyzing the nature of electronic structure in our systems. The original total DOS (TDOS) of our system was calculated based on eq. 6 and are shown in We have calculated the amount of charge on constituent $\mathrm{B}$ and $\mathrm{N}$ atoms in our systems by performing the Mulliken atomic charges.

The SWBNNT in the form of inner cylindrical are semiconductors due to the existence of an energy gap in the range of (250-310) KJ/mol (Tablel) which are between the valence bands and the conductor bands. The $(n, n)$ and $(m, n)$ single-walled boron nitrogen nanotubes (SWBNNTs) that have similar diameter. The partial density of states (PDOS) of different contributions of B and $\mathrm{N}$ are plotted in Fig 2-5 with the total DOS for comparison. The data for interaction energy shows that the $(3,3) @(7,7)$ DWBNNTs have a stable form comparing to other systems which yields a suitable charge transfer for the Nano capacitor.

The calculated values of charge transfer for $(3,3$ BNNTs @ $(7,7)$ CNNTs, $(3,3)$ BNNTs @ $(7,7)$ CNNTs and $(3,3)$ BNNTs @ $(7,7)$ CNNTs from inner to outer tubes is found to be $1.02,0.47$ and 1.22 electrons respectively, which is an acceptable value (Table2) and it is negligible for the $(5,5) @(12,12)$ structures due to their unstable forms. This analysis clearly indicates that a significant amount of charge is transferred from inner tubes which are closed to outer tubes. However the values of -q may scatter owing to the ambiguities in placing boundary between $\mathrm{Zn}$ and $\mathrm{O}$ in the structure. 
Table 2. $\Delta$ (stability energy) and charge distribution based on the geometry of $\mathrm{BN}(\mathrm{m}$, m)NTs@ C(n, n)NTs structures

\begin{tabular}{|l|l|l|l|c|}
\hline BNNTs @CNNTs & $\begin{array}{l}\operatorname{Ln}\left(\mathrm{R}_{\mathrm{OUT}} / \mathrm{R}_{\mathrm{i}}\right. \\
\mathrm{nn})\end{array}$ & $\sum \mathrm{Q}(+)$ & $\sum \mathrm{Q}(-)$ & $\Delta$ (stability energy) \\
\hline $\mathbf{( 3 , 3 ) @ ( 7 , 7 )}$ & 0.80 & +1.07 & -1.01 & $-4.19 \mathrm{eV}$ \\
\hline $\mathbf{( 4 , 4 ) @ ( 9 , 9 )}$ & 0.79 & 0.09 & -0.08 & $-0.99 \mathrm{eV}$ \\
\hline $\mathbf{( 4 , 4 ) @ ( 1 1 , 1 1 )}$ & 0.99 & 0.11 & -0.08 & $+0.35 \mathrm{eV}$ \\
\hline $\mathbf{( 5 , 5 ) @ ( 1 0 , 1 0 )}$ & 0.61 & 0.04 & -0.04 & $-0.60 \mathrm{eV}$ \\
\hline $\mathbf{( 5 , 5 ) @ ( 1 2 , 1 2 )}$ & 0.80 & 0.06 & -0.05 & $+11.00 \mathrm{eV}$ \\
\hline
\end{tabular}

Table3: Several DFT methods including two levels of of DFT/Semi empirical

\begin{tabular}{|c|c|c|c|c|c|c|c|}
\hline Methode & $\overline{\Sigma Q(\text { out })}$ & $\overline{\Sigma Q(i n n)}$ & $\begin{array}{l}\text { Fermi } \\
\text { energy }\end{array}$ & $\begin{array}{l}\text { Partial } \\
\text { charges }\end{array}$ & $\mathrm{HOMO}$ & LUMO & Gap \\
\hline M06HF/PM6 & -0.42 & +0.42 & -814.3 & \pm 0.47 & -0.31011 & -0.21500 & 249.6 \\
\hline M06/PM6 & -0.44 & +0.44 & -814.1 & \pm 0.46 & -0.310109 & 0.21500 & 249.5 \\
\hline M052x/PM6 & -0.47 & +0.47 & -814.4 & \pm 0.45 & -0.31011 & 0.21500 & 249.7 \\
\hline M062x/PM6 & -0.43 & +0.43 & -817.0 & \pm 0.62 & -0.31119 & 0.211091 & -262.6 \\
\hline M05/PM3MM & +0.03 & -0.03 & -890.6 & \pm 0.54 & -0.339219 & -0.07527 & 692.7 \\
\hline
\end{tabular}

Table4. The dielectric constant and capacitance $\mathrm{C}(\mathrm{g})$ for inner and outer BN dopant

\begin{tabular}{|c|c|c|c|c|c|c|}
\hline $\begin{array}{l}\text { SWBNTs@S } \\
\text { WCNTs }\end{array}$ & $\begin{array}{l}\mathrm{Ln}\left(\mathrm{R}_{\mathrm{OUT}} / \mathrm{R}_{\mathrm{i}}\right. \\
\mathrm{nn})\end{array}$ & $\sum \mathrm{Q}$ (out)(+) & $\sum \mathrm{Q}(\mathrm{inn})(-)$ & $\sum \mathrm{V}(2)-\mathrm{V}(\mathrm{l})$ & $\mathrm{C}(\mathrm{G})$ & $\mathrm{k}$ \\
\hline $\begin{array}{c}(3,3) @(7,7)^{*} \\
\text { (inner) }\end{array}$ & 0.82 & 1.22 & -1.22 & 2.3 & 9.29 & 1.37 \\
\hline $\begin{array}{c}(3,3) @(7,7) \\
\begin{array}{c}(3,3) @(7,7)^{*} \\
\text { (outer) }\end{array}\end{array}$ & 0.82 & 1.02 & -1.02 & 1.88 & 8.58 & 1.26 \\
\hline
\end{tabular}


The results are listed in tables 1-4, and the figures are explained in Figs 2-4. The geometry optimization for TWCNTs has been done with HF and B3LYP methods at different basis sets such as $4-31 G, 6-31 G, 6-31 G^{*}$ and $6-311 G^{*}$. Then thermodynamic properties were calculated for this structure in gas phase at $298.15 \mathrm{~K}$ in the same methods and basis sets. A comparison of Gibbs free energy (G), Enthalpy (H), Entropy (S) and Internal energy (E) in different methods and basis sets are shown in table 4. As shown in table 4, the maximum values for Gibbs free-energy $(\mathrm{G})$, Enthalpy $(\mathrm{H})$ and Internal energy $(\mathrm{E})$ were calculated when $6-311 \mathrm{G}^{*}$ basis set had been applied at B3LYP method.

HOMO and LUMO and Gap energy of TWCNTs (atoms between, 500-540) are listed in Table 1.

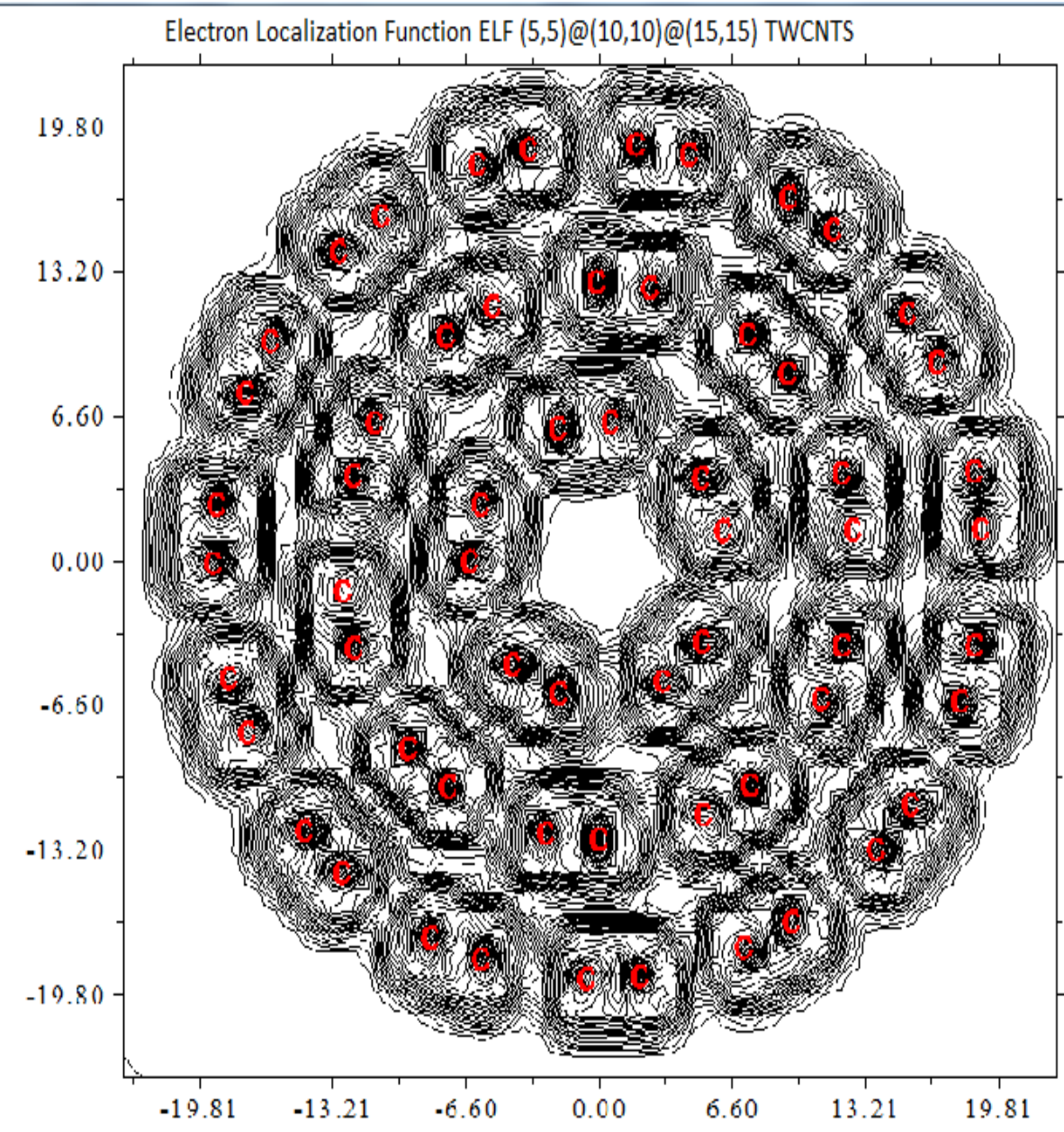




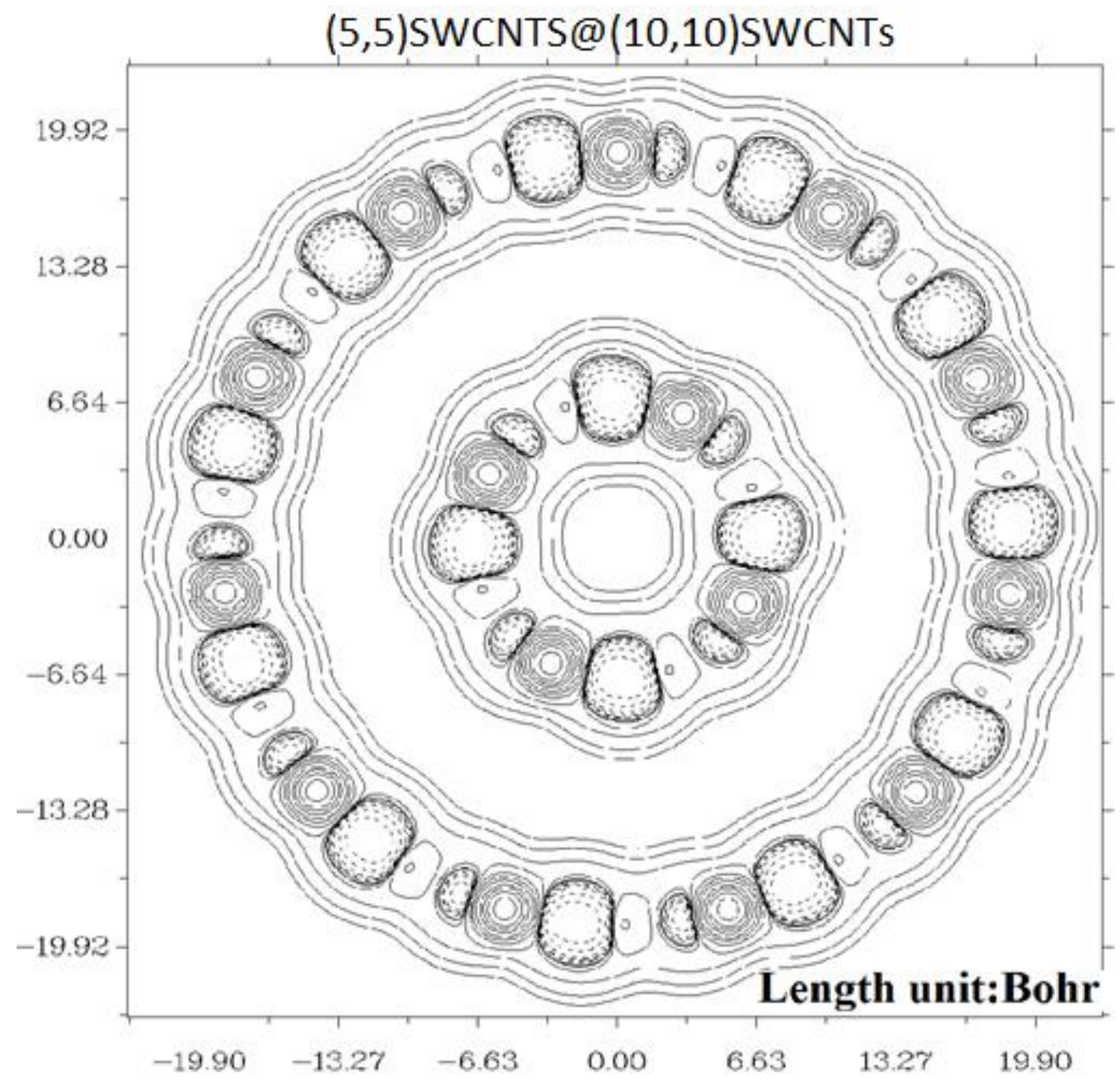

Fig3. ELF of $(5,5) @(10,10) @(15,15)$ TWCNTS

Out Put and plot of density electron from Atoms 1-540 are shown. Localized Orbital Locator (LOL)@Electron Localization Function ELF of $(5,5) @(10,10) @(15,15)$ TWCNTS are shown in Figs2-5. Relief map and Shaded Surface map with projection for electron density of $(5,5) @(10,10) @(15,15)$ TWCNTs in Fig3\& $\&$. According to the results that are shown in table2, the largest values have been obtained in B3LYP method. 
REVISTA DE LA UNIVERSIDAD DEL ZULIA. $3^{a}$ época. Año 11 N²9 29, 2020 Farhami and Monajjemi /// Investigation about electrostatic ...
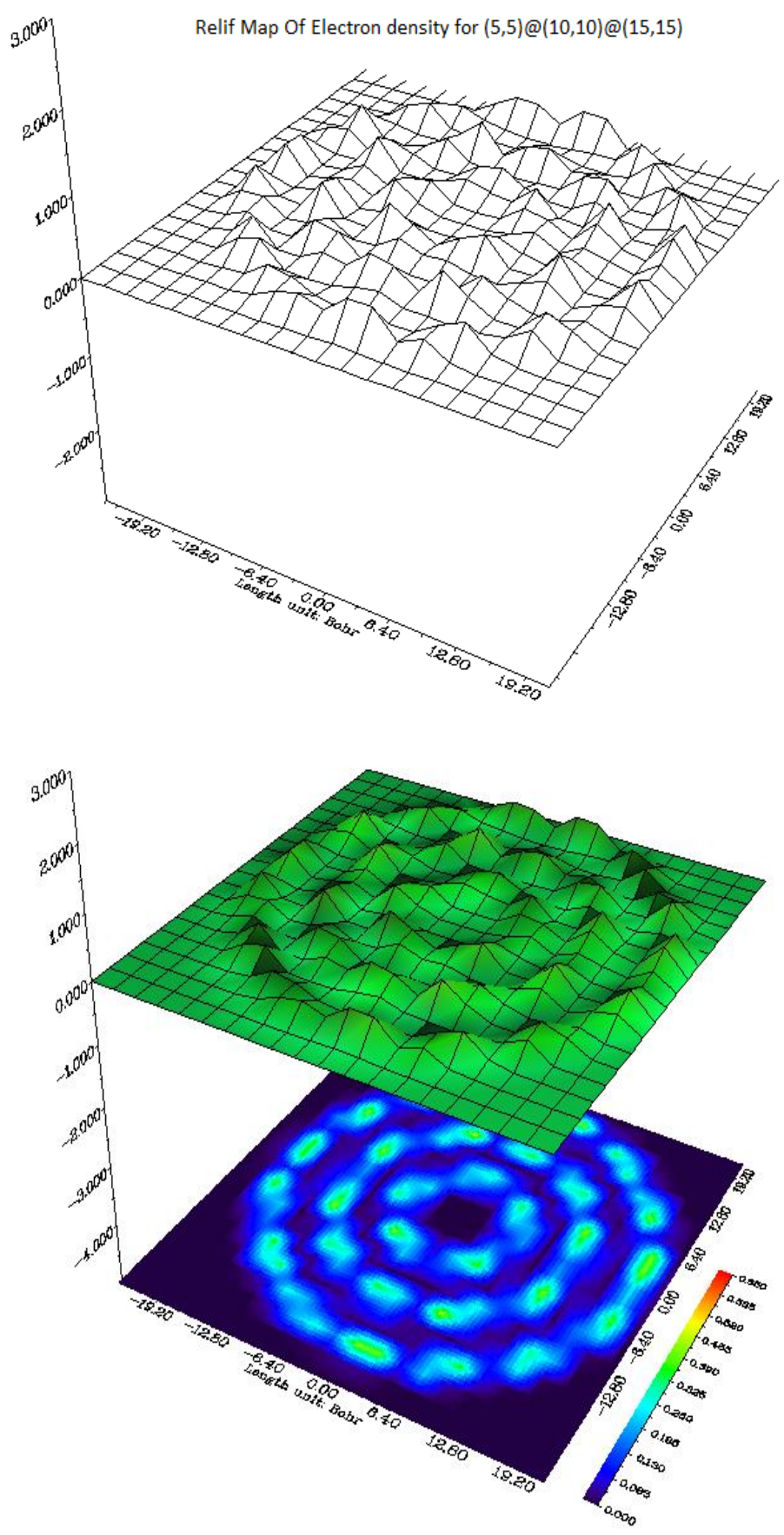

Fig. 4. Relief map and Shaded Surface map with projection for electron density of $(5,5) @(10,10) @(15,15)$ TWCNTs 
REVISTA DE LA UNIVERSIDAD DEL ZULIA. 3época. Año 11 N²9, 2020 Farhami and Monajjemi /// Investigation about electrostatic ...

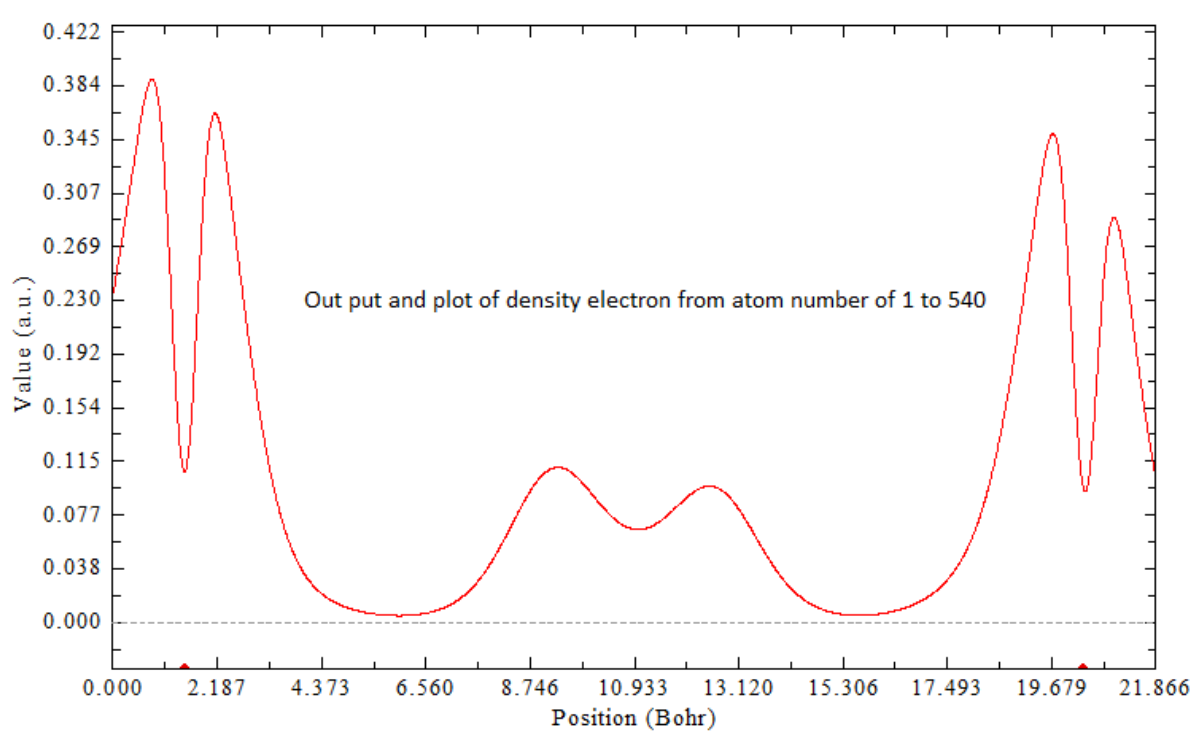

Fig 5. Out Put and plot of density electron from Atoms 1-540

Conclusion

The stability of TWCNTs and SWBNNTs @ DWCNTs were investigated. It is found that the amount of Gibbs free energy $(G)$, Enthalpy $(H)$ and internal Energy (E) obtained in DFT levels in the gas phase are the largest amount and also optimization of TWCNTs and SWBNNTs @ DWCNTs at the HF levels which means DFT calculation are more accurate and suitable for these Nano structures.

\section{References}

Bourgeois, L., Bando, Y., Han, W.Q., Sato, T. (2000). Structure of boron nitride nanoscale cones: Ordered stacking of $240^{\circ}$ and $300^{\circ}$ disclinations Phys. Rev. B, (61), 7686.

Derakhshandeh, Maryam (2015). NMR Shielding Tensors and Thermodynamic Investigation of TWCNTs including BN doping: A model for H2 storage. Oriental Journal of Chemistry, 31 (3), 1287-1297. 
Farhami, N., Monajjemi, M., Zare, K. (2017). Non Bonded Interactions in cylindrical capacitor of (m, n) @ (m', n') @ (m",n") Three Walled Nano Carbon Nanotubes. Oriental Journal of Chemistry (33) , 3024- 3030, http://dx.doi.org/10.13005/ojc/ 330640

Frackowiak, E., Béguin, F. (2002). Electrochemical storage of energy in carbon nanotubes. Carbon, (40), 1775-1787, https://doi.org/10.1016/S0008-223(02)00045-3

Iijima Sumio (1991). Synthesis of Carbon Nanotubes. Nature, (354), 56-58.

Lee, V.S., Nimmanpipug, P.,Mollaamin, F., Kungwan, N., Thanasanvorakun, S., Monajjemi, M.(2009). Investigation of single wall carbon nanotubes electrical properties and mode analysis: Dielectric effects. Russian journal of physical chemistry A (83), 2288-2296, https://doi.org/10.1134/S0036024409130184.

Lu, T., Chen, F. (2012). Multiwfn: A Multifunctional Wavefunction Analyzer. J. Comp. Chem., 33, 580-592, https://doi.org/10.1002/jcc.22885.

Madani, M.S., Monajjemi, M., Aghaei, H. (2017). The Double Wall Boron Nitride Nanotube: Nano-Cylindrical Capacitor, Oriental Journal of Chemistry , (33) , 12131222 , http://dx.doi.org/10.13005/ojc/330320.

Marín, J.; Fernández, K.; Behling, E.; Carrasquero, S.; Angulo, N. (2019). Características de la biopelícula de un reactor RBC en presencia y ausencia de metales pesados, Revista de la Universidad del Zulia, 10 (26), 113-141.

Monajjemi, M., Chegini, H., Mollaamin, F., Farahani, P. (2011). Theoretical Studies of Solvent Effect on Normal Mode Analysis and Thermodynamic Properties of Zigzag $(5,0)$ carbon nanotube. Fullerens Nanotubes carbon and nanostructures (19), 469-482, https://doi.org/10.1080/1536383X.2010.494783. 